\title{
Communication Tools in Offshore Development with Scrum
}

\author{
F. Sahar, S. T. Raza, and M. N. Nasir
}

\begin{abstract}
Offshore development with Scrum is a progressively more important development approach for software development organization as it fetches attractive opportunities. Offshore development is already facing several problems and Scrum method brings more challenges in the form of their reliance of verbal communication. The aim of this paper is to present a research design for providing recommendation for rich communication system in offshore development with Scrum. It discusses the research methodology used for developing the recommendation for rich communication system.
\end{abstract}

Index Terms-Communication tools, development, offshore, scrum.

\section{INTRODUCTION}

Offshore development with Scrum has become a model in modern software industry [1]. In offshore development teams are distributed across the globe and used communication tools in order to communicate with stakeholders [2]. The main factors behind offshoring are: cost, time to market, access to skill and cheaper labor, and market proximity and these factors have motivated the software development effort towards a globally distributed environment [2], [3]. Simultaneously, many studies show that offshore development with Scrum is risky. It has been found that projects take $2.5 \%$ more time to complete as compared to onsite development because of communication related issues [4]. A recent survey by [5] describe that success percentage of co-located team is $83 \%$, distributed but physically reachable is $72 \%$ and distributed across the globe is $60 \%$ [5]. The research on offshore development with Scrum shows that communication is major issues [2], [6]-[8].

This paper describes the design of research for developing recommendation for rich system in offshore development with Scrum. The paper is divided into five sections. First section describes the introduction, background and related work. The second section provides purpose of this study. Third section describes research questions and motivation for these research questions. Section four consists of two parts. First part describes qualitatively research method and second part describes the quantitative research method. Last section provides conclusion.

\section{A. Background}

In offshore development, organizations relocate all or part of their development activities into another country with their own affiliates [3]. Although offshore development become a

Manuscript received January 9, 2013; revised April 19, 2013.

The authors were with Blekinge Institute of Technology, SE- 37179 Karlskrona, Sweden (e-mail: farrukhsahar@yahoo.com, tosif@live.com, Nadeem.nasir@live.com). norm and it is increasing day by day but software organizations facing some challenges like: communication, Time difference, cultural difference, and geographical difference [9].

Scrum is an agile method and has become a de-facto standard in software development organizations [9]. It is a process skeleton that consists of set of practices, roles and documents [10], [11]. The Scrum team has three roles and these are Scrum master, Product owner, and development team. The set of practices consist of Scrum meetings like daily Scrum, Sprint planning meeting, Sprint review meeting. The Scrum documents consist of Product backlog, Sprint backlog and Burn down chart [10], [11]. The success factors of Scrum are collocated development, verbal communication; focus on team building and collaboration [11].

The problem with Scrum method is when used in offshore development that in offshore development, team members are thousands of miles apart from each other. Scrum demand close communication between stakeholders [10], [11]. This communication suffers when team members are away from each other. For example, normally in offshore development process, project' $\mathrm{s}$ development part is done in one country and clients lead the project as Product owner or Scrum Master in another country. They have to communicate with each other on daily basis as required by Scrum. So Success ratios of offshore project depend upon communication. Communication is a major challenge in offshore development and it creates hurdles in the success of offshore development project. There is need to increase communication between stakeholders. Communication tools act as a central repository of Scrum communication in offshore development. It enables Scrum roles to communicate with and access the Scrum documents at dispersed locations. For example in order to meet the demand of rapid change in requirements Scrum required continuously communication with customers / clients. This rapid change in requirements can only be successful, if everybody hear about the change and it is possible to transfer complete / unambiguous information between team members. This is only possible with the use of efficient communication tools in offshore development with Scrum.

\section{B. Related Work}

In Offshore development teams are dispersed at geographically boundaries. It is suitable for organizations of all size and these days it becomes a common practice [1], [7]. The [2], [3], [7] provides main reasons for offshore development. Although these reasons provide attractive opportunities for software development organizations, offshore development is facing same problems as co-located development. Communication, software quality, requirement engineering, problems with schedule and budget are common issues and these issues become more verse in offshore development [7]. 
In order to overcome these issues several tools are used. These tools are: voice communication, videoconference, instant messaging, emails and whiteboards [4], [7], [12]. Communication play an important role in the success of an offshore development project and it become more important in Scrum because of its nature and structure [4], [7].

In [7], [13] highlights the communication challenges, and its support for offshore development But it hardly addresses need of communication tools, communication tools challenges and its need in offshore development with Scrum.

As [14] provides problems that arise in requirement engineering phase, designing phase, development phase of software development because of poor communication between stakeholders in offshore development with Scrum. Some articles like [11] provide a simple way for adaption of Scrum in an offshore development. "The five Dysfunction of a team" provides a simple way for establishing, management and growing an offshore team [15]. This model is easy to understand but difficult to do [15]. The [16] discuss outsource project management with distributed Scrum

The [10] discuss how to achieve and keep hyper productive state in offshore development. The researcher and organizations are trying to find the answers that how to maintain and achieve same velocity as localized team [10]. The [17] discuss the importance of communication tools in offshore development and conclude that a good tool provide big impact on communication and success of offshore development project.

Expected key contribution of the study, in the area of communication tools in offshore development with Scrum are:

- Providing a systematic literature review of communication tools in offshore development with Scrum.

- Finding suitable tools for communication in offshore development with Scrum.

- Finding the challenges of communication tools in literature and in industry.

- Categorization of effectiveness and ineffectiveness of communication tools in offshore development with Scrum.

- Design the recommendation for rich communication system in offshore development with Scrum.

- Ensuring that the proposed recommendations are valid for industry.

\section{Qualitative Approach}

1) Data collection: For data collection, we used two qualitative methods i.e. systematic literature review and interviews.

Systematic literature review: a systematic literature review is conducted for getting an idea of the state of art approaches. This systematic literature review is performed under the guidelines of [18]-[20]. A comprehensive systematic review protocol is designed for selection of primary and secondary studies, appraisal, study inclusion/ exclusion criteria, quality assessment and for synthesis.

The need for systematic literature review: The objective of this systematic literature review is to summarize the work done in communication tools that are used by Scrum in offshore development during the years of 2000-2009. This systematic literature review provides: a way to understand the research in the area of communication in offshore development with Scrum, a description of communication tools that are used in industry in offshore development, list of communication challenges that arise due to the used of poor communication tools and categorization of effective and ineffective tools. This provides help in designing the recommendation for establishing rich communication in offshore development with Scrum.

Systematic literature review protocol: A systematic literature review protocol provides a way to conduct the systematic literature review. This systematic literate review provides the answers of RQ1 and provides background knowledge for interviews. More detail about our review protocol is available in assignment 1 .

Interviews: Interviews are useful for collection of historical data from interviewee, opinion about specific domain and for getting unknown information [21]. In this study interviews are used to know the answers of RQ2 and to know the state of practice approach. Interviews are organized in semi structure way. The reason for semi structure interviews is that they involve both open and close ended questions [13]. Interviews with Scrum Master, Software developers, and Software Tester are performed.

The aim of this interview is to find, understand the difference and commonalities between state of art and state of practices approach.

Interview Design: Interviews are used in this research in order to collect data about communication tools and communication challenges in offshore development with Scrum.

Data Collection and Analysis: During the interview data was collected by listening, writing and recording the interview answers. As four researches participated during interview, one researcher noted down the important points, second recorded through recording device, third and fourth communicates with interviewee. Data was analyzed by understanding and interpretation of collected data.

Interview Execution Planning: Interviews were organized at the site of interviewee. The normal duration of interview is one hour. Interview goals, background of research and some questions are sent to interviewee before the interview date. At start of interview the purpose of interview and problem under discussion are briefly explain to interviewee.

Industrial interviews Execution: Interviews were executed with two organizations of Sweden. Research background, and some interview questions were send to interviewee by email before the interview date. On the interview day, first we gave brief introduction of research, topic, purpose of interviews, and introduction of participants. After that we explain the purpose of recording the interview and take permission so that we can record the interviews. It was make sure that we discard the audio recording after analysis. Then interviewee briefly introduced themselves and formal interview started.

2) Expected Outcome: The aim of this study is to design the recommendation for rich communication system between stakeholders in offshore development with Scrum. The main outcomes of the qualitative research are as: 
Systematic reviews results in understanding of state of art approaches of communication tools, Scrum, and offshore development. The communication tools those are useful in offshore development with Scrum. The challenges of communication tools is in literature and industry.

\section{Quantitative Approach}

The experiment identifies effective tools in offshore development with Scrum. The subjects were categorized the tools on the basis of effectiveness. For this purpose a form is given to subjects and they write information under four classification and these are: video conference, voice communication on Skype, instant messages and email.

The experiment design is based upon [22] and given below:

1) Definition. Object of study: The objects studies are: communication tools that are: Video conference, Voice communication on Skype, instant messages and email.

2) Purpose: The purpose of this experiment is to categorize the communication tools into effective and ineffective tools in offshore development with Scrum.

Quality Focus: The quality focus is the effectiveness of four communication tools in offshore development with Scrum.

Perspective: The experiment is executed from researcher and organization perspective. From researcher perspective it is used to categorize the communication tools into effective and ineffective with respect to offshore development with Scrum. From organization perspective the results of experiments are useful to improve communication in offshore development with Scrum.

Context: The experiment is executed with the help of one Scrum master and four software developers. These practitioners belong to offshore development with Scrum and involve in communication in offshore development with Scrum. The experiment is executed in the industry. The participants are communicating on communication tools in offshore development with Scrum.

Summary of definition: The experiment definition is as follow:

- Analyze communication tools (voice communication on Skype, instant messages and email)

- For the purpose of evaluation.

- With respective to effectiveness.

- From the point of view of the researcher.

- In the context of industry experts of communication tools in offshore development with Scrum.

- Performing communication over a project.

3) Planning Context Selection: The context of this experiment is the effectiveness of four different communication tools used in offshore development with Scrum for communication between stakeholders at different locations. Following lines will describe the characteristics of this experiment.

Online- This experiment is performed in real time environment. For this reason, a prior contacted organization was to ensure that suggestions will be provided to them after the successful completion of experiment for improvement of their communication system. This will makes sure that participants perform this experiment in a professional way.
The instruments used in this experiment are available and taken from a real project that is already running. The subjects already have experience of using these communication tools. On the basis of this information maximum time required for experiment is estimated and set.

Subject- The subjects of this experiment are software developers and Scrum Master.

Real- The finding and results of this experiment can be useful for Scrum Master and for Project Managers in software development organization.

General- The communication tools (Video conference, voice chat, instant messages and email) are used in the organization working offshore development with Scrum. The results of this experiment may be applied to other organizations working with offshore development with Scrum.

Measures needed: Performance of each communication is check through time required to transfer information about one software requirement, quality of communication, and understanding of requirement by the recipient.

Variables Selection: The independent variables for this experiment are given below:

Communication tool, i.e. which communication tools is being used for communication.

Age of participants and Operating systems

The dependent variables for this experiment are given below: The dependent variable is performance of communication tools, communication cost, accuracy of voice and text. Performance of communication tools is measured by time taken to understand the requirement, time taken for establishment of communication link. Communication cost is measured by cost of internet, cost of time of subject and Scrum Master, cost of electricity and cost of equipment.

Selection of Subjects: Those software developers and Scrum Master are selected as a subject of this experiment which takes part in offshore development with Scrum. Those peoples which communicate with offshore development on regular basis are selected as a subject of this experiment.

4) Experiment design: The design of experiment is as follow:

Randomization: The subjects are selected randomly in a sense that software developers have different background, experience, skills and working in offshore development. We have to make sure that equally numbers of software developers perform this experiment on video conference, voice communication, instant messages, and email.

Blocking: The subjects selected for this experiment have different experience with offshore development with Scrum which ranges between one to five years. However, we do not apply any systematic approach for blocking because they have to write about the effectiveness of communication tools in offshore development.

Balancing: The experiment is balanced because same number of subjects done communication through video conference, voice communication, instant messages, and email.

Design Type: The hypotheses for the evaluation describe that the design is: one factor with more than two treatments. The factor is communication tools and treatments are video conference, voice communication, instant messages and e-mail. According to [3] ANOVA test (parameter test) is 
appropriate if the division of collected data is Gaussian and Kruskal-Wallis test (non-parameter test) if the distribution is not normal.

5) Instrumentation: Following objects are used during the experiments:

- Each subject will have access to a computer that is connected to internet and all communication tools are available.

- A simple software requirement that is used for communication between subjects.

- Communication tools will be given to all subjects. This means that $1 / 4$ will get Video conference, $1 / 4$ will get voice communication, $1 / 4$ instant messages and $1 / 4$ will get email.

- A manual form will be distributed between all subjects to write down the time spend in performing the activity.

- The researchers will use checklist in order to find the issues that arise in communication for each communication tools.

6) Experiment Execution. Preparation: Experiment is executed in a separate lab for software development in each organization. All the equipments that are necessary are already available from organization side. The date and time of experiment was announced and informed to the participants in advance. All the instruments are tested one day before the experiment to avoid any abnormal situation.

Execution: The experiment is executed on time. First a brief overview of experiment is given to each participant and then a short question and answers session was organized to remove ambiguity. All the researchers were available during the experiments and helped participants as according to their need.

Data Collection: At the end of experiments, the subject handed over the results to researchers. Data was collected in text format from all the groups performing communication on videoconference, voice chat, instant messages and emails. At the end we also write the remarks of subject about over all experiment execution.

Data Validation: In order to validate data a check list [23] are used. This check list is consist of questionnaires about the experiments and was checked by the subjects. This method was to ensure subject role in experiment and treatment that applied.

7) Analysis and Interpretation: Analysis of data is performed under the guidelines of [21]. This procedure defines following step for data analysis and interpretation.

Descriptive statistics: It is used to get an overview of data set distribution [21].

Data set reduction: It is used to improve the quality of data set by removal of low quality data points that were used with statistical methods [21].

Hypotheses testing: Disprove the Null hypothesis with statistical methods [21].

\section{CONCLUSION}

The paper has two fold contributions in the area of communication tools in offshore development with Scrum.
First it provides the systematic literature review of said topic. Secondly it has proposed the recommendation for rich communication system in offshore development with Scrum. It discusses the research design for developing the recommendation from systematic literature review, interviews and experiment. The experiments results are used to categorized the communication tools into effective and ineffective tools. The paper also contributes in selection of research methodology to be use for this kind of problems.

\section{REFERENCES}

[1] C. Wohlin, M. Höst, P. Runeson, C. Ohlsson, and A. Wesslén, Experimentation in Software Engineering: An Introduction, Kluwer Academic Publishers Norwell, MA, USA, 2000.

[2] M. F. Nisar and T. Hameed, "Agile methods handling offshore software development issues," in Proc. 2004. 8th International Multitopic Conference, 2004. INMIC, Lahore Pakistan, 2004, pp. 417-422.

[3] A. Dutta and R. Roy, "Offshore outsourcing: counteracting forces and their dynamic effects," in Proc. 38th Annual Hawaii International Conference on System Sciences, HICSS '05., Hawaii, 2005, pp. 258a.

[4] J. Herbsleb and D. Moitra, "Global software development," IEEE Software, vol. 18, pp. 1620, January/February 2001.

[5] J. Herbsleb and A. Mockus, "An empirical study of speed and communication in globally distributed software development," IEEE Transactions on Software Engineering, vol. 29, pp. 481-494, January 2003.

[6] V. Hazrati. Results of agile adoption survey 2008. [Online]. Available: http://www.infoq.com/news/2008/05/agile-adoption-survey-2008.

[7] Seminar in Scandinavia, IRIS 29 - Paradigms Politics Paradoxes, August 12-15, Helsingoer, Denmark, 2006.

[8] L. Layman, L. Williams, D. Damian, and H. Bures, "Essential communication practices for extreme programming in a global software development team," Information and Software Technology, vol. 48, pp. 781-794, Sept.2006.

[9] E. Therrien, "Overcoming the challenges of building a distributed agile organization", in Proc. Agile Development Conference, AGILE 2008, Toronto, Canda 2008, pp. 368-372.

[10] J. Sutherland, G. Schoonheim, E. Rustenburg, and M Rijk, "Fully distributed scrum: the secret sauce for hyperproductive offshored development teams," in Proc. Agile Development Conference, AGILE 2008 , Toronto, Canda 2008, pp. 339-344.

[11] S. H. Rayhan, N. Haque, "Incremental Adoption of Scrum for Successful Delivery of an IT Project in a Remote Setup," in Proc. Agile Development Conference, AGILE 2008, Toronto, Canda 2008, pp. 351-355.

[12] T. Schümmer and J. Schümmer, Support for Distributed Teams in Extreme Programming, 1st ed. G. Succi and M. Marchesi, Eds. Addison-Wesley Longman Publishing, 2001.

[13] J. Sauer, "Agile practices in offshore outsourcing - An analysis of published experiences," in Proc. 29th Information Systems Research, Elsinore, Denmark, 2006.

[14] M. Kircher, P. Jain, A. Corsaro, and D. Levine, "Distributed extreme programming," in Proc. XP2001, Sardinia, Italy, May 2001, pp. 66-71.

[15] G. Fischer, "Social creativity: Turning barriers into opportunities for collaborative design," in Proc. Eighth Conference on Participatory Design, New York, USA, pp. 152-161, 2004.

[16] E. Uy and N. Ioannou, "Growing and sustaining an offshore scrum engagement," in Proc. Agile Development Conference, AGILE 2008, Toronto, Canda 2008, pp. 345-350.

[17] J. Sutherland, A. Viktorov, J. Blount, and N. Puntikov, "Distributed scrum: Agile project management with outsourced development teams," in Proc. 40th Annual Hawaii International Conference on System Sciences, 2007. HICSS 2007, Hawaii, 2007, pp. 274a.

[18] C. Ken, Agility and Requirements, Advanced Technology Systems Corporation, Sept 10th, 2008.

[19] J. E. Hannay, D. I. K. Sjoberg, andT.Dyba, "A systematic review of theory use in software engineering experiments," IEEE Transactions on Software Engineering, vol. 33, no. 2, pp. 87-107, Feb. 2007.

[20] B. Kitchenham and S. Charters, "Guidelines for performing systematic literature reviews in software engineering," Software Engineering 
Group, School of Computer Science and Mathematics, Keele University, EBSE Technical Report Version 2.3, Jul 2007.

[21] T. Dyba, T. Dingsoyr, and G. K. Hanssen, "Applying systematic reviews to diverse study types: an experience report," in Proc. First International Symposium on Empirical Software Engineering and Measurement, 2007. ESEM 2007, Madrid, Spain, Sept. 2007, pp. 225-234.

[22] C. Seaman, "Qualitative methods in empirical studies of software engineering," IEEE transactions on Software Engineering, vol. 25, no. 4, pp. 557-572, 1999.

[23] J. Cresswell, Qualitative Inquiry and Research Design: Choosing Among Five Traditions, Sage publications, London, 2006.

Farrukh Sahar was with Blekinge Institute of Technology, SE- 37179 Karlskrona, Sweden. (e-mail: farrukhsahar@yahoo.com).

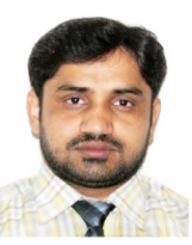

Syed Tosif Raza was with Blekinge Institute of Technology, SE- 37179 Karlskrona, Sweden. He is now with the World Health Organization, Regional Office for Europe Copenhagen (e-mail: tosif@live.com)

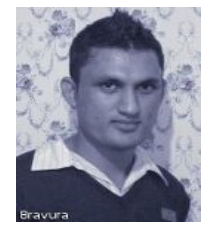

Muhammad Nadeem Nasi was with Blikinge Institute of Technology, SE- 37179 Karlskrona, Sweden (e-mail: Nadeem.nasir@live.com). 